Approximate Analysis of Discrete-Time Tandem Queueing Networks with Bursty and Correlated Input Traffic and Customer Loss

Dooyeong Park

Harry G. Perros

Hideaki Yamashita

Center for Communications and Signal Processing Department of Computer Science North Carolina State University

TR-92/19

October 1992 


\title{
Approximate Analysis of Discrete-time Tandem Queueing Networks with Bursty and Correlated Input Traffic and Customer Loss
}

\author{
Dooyeong Park \\ Department of Eletrical and Computer Engineering, and \\ Center for Communications and Signal Processing \\ North Carolina State University \\ Raleigh, NC 27695 \\ Harry G. Perros \\ Department of Computer Science, and \\ Center for Communications and Signal Processing \\ North Carolina State University \\ Raleigh, NC 27695 \\ Hideaki Yamashita \\ Department of Mechanical Engineering \\ Sophia University \\ Kioi-cho 7-1, Chiyoda-ku \\ Tokyo 102, Japan
}

Abstract- We first describe an approximation method for fitting a two-state MMBP to the departure process of a two-state MMBP/Geo/1/K queue. The flted MMBP is shown to have a good accuracy. The model for fitting a two-state MMBP is then used in a simple decomposition algorithm to analyze a tandem conflguration of flnite capacity queues with customer loss. The decomposition algorithm is validated by comparing it against simulation and it is shown to have a good accuracy. 


\section{Introduction}

Discrete-time single server queues with or without finite capacity have been extensively analyzed. For a review of relevant results see Pujolle and Perros [1]. However, little has been done for the analysis of networks of discrete-time finite capacity queues.

In this paper, we analyze a tandem queueing network of discrete-time finite capacity queues with customer loss. The arrival process to the first queue is assumed to be a twostate Markov Modulated Bernoulli Process (MMBP) and the service times at each queue are assumed to be geometrically distributed. Queueing networks of discrete-time finite capacity queues with customer loss do not lend themselves to an exact analysis. However, they can be analyzed approximately using the notion of decomposition. That is, the network is decomposed into individual queues, and each queue is then analyzed separately. The most important aspect of such a decomposition is the characterization of the arrival process to an intermediate queue. In continuous-time queueing networks, typically, such as an arrival process is characterized approximately by a two-phased Coxian distribution, or by a more general phased-type distribution. Also, it has been characterized by a general distribution defined by the mean and squared coefficient of variation. In this paper, we first describe an approximate model for fitting a two-state MMBP to the departure process of a twostate $\mathrm{MMBP} / \mathrm{Geo} / 1 / \mathrm{K}$ queue. The fitted MMBP is shown to have a good accuracy. This fitting model is then used in a simple decomposition algorithm to analyze a tandem queueing network with discrete-time finite capacity queues with customer loss. Validation tests showed that the decomposition algorithm has a good accuracy.

The problem of fitting a discrete-time distribution to the departure process of a discretetime queue is relatively unexplored. Park and Perros [2] approximated the departure process of an IBP/Geo/1/K queue by an IBP. Meier-Hellstern [3] developed an iterative algorithm for fitting two-state MMPP to observational data using maximum likelihood estimation. The interdeparture distribution of a discrete-time GI/G/1 queue has been obtained by TranGia [4] using the Fast Fourier Transform. Ohba, Murata and Miyahara [5] analyzed a 
discrete-time single-server queue with three different arrival streams and they obtained the departure process. Discrete-time queueing networks have been analyzed by Morrison [6], Hsu and Burke [7], Ohba, Murata, and Miyahara [5] and Pujolle [8] assuming infinite capacity queues. Bhargava et al [9] and Bocharov and Albores [10] analyzed tandem configurations of finite capacity queues with customer loss, but in continuous-time. Finally, several discretetime queueing networks of finite capacity queues with blocking have recently been analyzed in connection with the performance modelling of switch architectures for high-speed networks (see Morris and Perros [11] and the references within).

This paper is organized as follows. In section 2, we give a brief description of the twostate MMBP. The generating function of the interdeparture time of an MMBP/Geo/1/K queue is obtained in section 3 . In section 4 , we present a fitting model for characterizing the departure process as a two-state MMBP and examine its accuracy. In section 5 , we analyze a tandem configuration of finite capacity queues with customer loss using the fitting model described in section 4 . Finally, the conclusions are given in section 5 .

\section{The Two-State Markov Modulated Bernoulli Process}

A two-state MMBP is a Bernoulli process whose rate varies according to a two-state Markov chain. It captures the notion of burstiness and the correlation of successive interarrival times, which are two important characteristics of the anticipated traffic on future high-speed networks. It is characterized by the transition probability matrix $\mathbf{P}$ and by $\boldsymbol{\Lambda}$ given by:

$$
\mathbf{P}=\left[\begin{array}{cc}
p & \bar{p} \\
\bar{q} & q
\end{array}\right] \quad \text { and } \quad \Lambda=\left[\begin{array}{cc}
\alpha & 0 \\
0 & \beta
\end{array}\right]
$$

where $0<p, q<1, \bar{p}=1-p$, and $\bar{q}=1-q$. In a two-state MMBP, there is a geometrically distributed period of time (known as state 1) during which arrivals occur in a Bernoulli fashion with a specific probability $\alpha$. This period is followed by another geometrically distributed period (known as state 2) during which arrivals also occur in a Bernoulli fashion but with a different probability $\beta$. These periods alternate continuously. Given that the process is in the state 1 (or state 2 ) at slot $i$, it will remain in the same state in the next slot $i+1$ with 
probability $p$ (or $q$ ), or will change to the state 2 (or state 1 ) with probability $\bar{p}$ (or $\bar{q}$ ).

Let $t_{n}$ be the time interval between the $(n-1)$ st and $n$th arrival. It can be shown that the average arrival rate, i.e. the probability that any slot contains a cell, $\rho$ and the squared coefficient of variation of the interarrival time between successive arrivals, $C^{2}$ are as follows:

$$
\begin{aligned}
\rho & =\frac{1}{E\left\{t_{n}\right\}}=\frac{\bar{q} \alpha+\bar{p} \beta}{\bar{p}+\bar{q}} \\
C^{2} & =\frac{2 \rho\left[(\bar{p}+\bar{q})^{2}+(\bar{p} \alpha+\bar{q} \beta)(p+q-1)\right]}{(\bar{p}+\bar{q})[\bar{q} \alpha+\bar{p} \beta+\alpha \beta(p+q-1)]}-\rho-1 .
\end{aligned}
$$

Let $\pi$ be the stationary probability vector satisfying $\pi P=\pi$. Then, it can be shown that (see [12])

$$
E\left\{t_{n} t_{n+i}\right\}=\overrightarrow{\mathbf{p}}_{\mathbf{a}}(\mathbf{I}-\mathbf{M})^{-2} \mathbf{P} \Lambda \mathbf{T}^{i-1}(\mathbf{I}-\mathbf{M})^{-2} \mathbf{P} \vec{\lambda} .
$$

where $\mathbf{M}=\mathbf{P}(\mathbf{I}-\mathbf{\Lambda}), \vec{\lambda}=[\alpha, \beta]^{T}, \overrightarrow{\mathbf{p}}_{a}=\pi \mathbf{\Lambda} / \pi \vec{\lambda}$, and $\mathbf{T}=[\mathbf{I}-\mathbf{M}]^{-1} \mathbf{P} \mathbf{\Lambda}$. The autocorrelation coefficient of the interarrival time of a two-state MMBP for lag $i, \psi(i)$, can then be obtained, since $\psi(i)=\left[E\left\{t_{n} t_{n+i}\right\}-E^{2}\left\{t_{n}\right\}\right] / \operatorname{Var}\left\{t_{n}\right\}$. In particular, the lag 1 autocorrelation, $\psi(1)$, is given by

$$
\psi(1)=\frac{\alpha \beta(\alpha-\beta)^{2} \bar{p} \bar{q}(p+q-1)^{2}}{C^{2}(\bar{p}+\bar{q})^{2}[\bar{q} \alpha+\bar{p} \beta+\alpha \beta(p+q-1)]^{2}} .
$$

Of interest is also the autocorrelation coefficient of the number of arrivals of a two-state MMBP for $\operatorname{lag} i, \phi(i)$. This is given by (see [13]):

$$
\phi(i)=\frac{\bar{p} \bar{q}(\alpha-\beta)^{2}(p+q-1)^{i}}{(\bar{q} \alpha+\bar{p} \beta)[\bar{q}(1-\alpha)+\bar{p}(1-\beta)]} .
$$

\section{The Departure Process of a two-state MMBP/Geo/1/K Queue}

We consider a two-state $\mathrm{MMBP} / \mathrm{Geo} / 1 / \mathrm{K}$ queue, where the service time is defined over a slotted time axis. A service always starts at the beginning of a service slot, and the service time is assumed to be a geometrically distributed number of service slots with parameter $\sigma$. Service completion is assumed to take place just before the end of a service slot. The arrival process is also defined over a slotted time axis with the same slot size, and it is assumed to be a two-state MMBP with parameters $p_{A}, q_{A}, \alpha_{A}$, and $\beta_{A}$. The boundaries of the slots 
of the arrival process are assumed to be in-between the boundaries of the service slots. An arriving customer to an empty queue cannot start its service until the beginning of the next service slot, even though the server is free at the instant of its arrival. We examine this queue at the boundary of each service slot. The state of this queue can be described by the variables $(s, n)$. Variable $s$ represents the state of the arrival process and it takes the values: 1 if the arrival process is in the state 1,2 if the arrival process is in the state 2. Variable $n$ indicates the number of customers in the system including the customer in service. We have $n=0,1, . ., \mathrm{K}$, where $\mathrm{K}$ is the capacity of the system. Let $\mathbf{P}_{d}$ be the transition probability matrix of the queue. We can see that $\mathbf{P}_{d}=\mathbf{P}_{w d}+\mathbf{P}_{w o d}$, where $\mathbf{P}_{w d}, \mathbf{P}_{w o d}$, is a matrix that contains transitions with a departure respectively without a departure. Below, we first compute the generating function of the probability distribution of the interdeparture time, and then we obtain two different types of autocorrelation related to the departure process.

\subsection{The Generating Function of the Interdeparture Time Distribution}

Let $I$ be a random variable representing the time elapsing from the moment a departure occurs to the end of a service slot during which an arrival occurs. Also, let $S$ and $D$ be random variables representing the service time and the interdeparture time, respectively. The server idle period, $I$ can be expressed by

$$
I= \begin{cases}0 & 1-P^{+}(1,0)-P^{+}(2,0) \\ I_{1} & P^{+}(1,0) \\ I_{2} & P^{+}(2,0)\end{cases}
$$

where $I_{1}, I_{2}$, is a random variable indicating the time elapsing from the moment the system is in state $(1,0)$, respectively $(2,0)$, to the end of the service slot during which an arrival occurs. $P^{+}(s, n)$ is the probability that immediately after a departure the system is in the state $(s, n)$. Therefore, $I_{1}, I_{2}$, and $P^{+}(s, n)$ are given as follows:

$$
\begin{aligned}
& I_{1}=\left\{\begin{array}{cl}
1 & p_{A} \alpha_{A}+\bar{p}_{A} \beta_{A} \\
1+I_{1} & p_{A}\left(1-\alpha_{A}\right) \\
1+I_{2} & \bar{p}_{A}\left(1-\beta_{A}\right)
\end{array}\right. \\
& I_{2}=\left\{\begin{array}{cl}
1 & q_{A} \beta_{A}+\bar{q}_{A} \alpha_{A} \\
1+I_{1} & \bar{q}_{A}\left(1-\alpha_{A}\right) \\
1+I_{2} & q_{A}\left(1-\beta_{A}\right)
\end{array}\right.
\end{aligned}
$$




$$
\overrightarrow{\mathbf{P}}^{+}=\left[P^{+}(1,0), P^{+}(2,0), \cdots, P^{+}(1, K), P^{+}(2, K)\right]=\frac{\mathbf{x} \mathbf{P}_{w d}}{\mathbf{x} \vec{\lambda}_{d}}
$$

where $\bar{p}_{A}=1-p_{A}, \bar{q}_{A}=1-q_{A}, \vec{\lambda}_{d}=[0,0,1-\sigma, \cdots, 1-\sigma]^{T}$, and $\mathbf{x}$ is the steady state probability vector satisfying $\mathbf{x} \mathbf{P}_{d}=\mathbf{x}$. The generating function of the server idle period, $I(z)$, is then given by

$$
I(z)=1-P^{+}(1,0)\left[1-I_{0}(z)\right]-P^{+}(2,0)\left[1-I_{1}(z)\right]
$$

where $I_{1}(z)$ and $I_{2}(z)$ are the $z$-transforms of $I_{1}$ and $I_{2}$, respectively.

$$
\begin{aligned}
& I_{1}(z)=\frac{\left(1-\beta_{A}\right) \alpha_{A}\left(\bar{p}_{A}-q_{A}\right) z^{2}+\left[p_{A} \alpha_{A}+\bar{p}_{A} \beta_{A}\right] z}{\left(1-\alpha_{A}\right)\left(1-\beta_{A}\right)\left(p_{A}-\bar{q}_{A}\right) z^{2}-\left[q_{A}\left(1-\beta_{A}\right)+p_{A}\left(1-\alpha_{A}\right)\right] z+1}, \\
& I_{2}(z)=\frac{\left(1-\alpha_{A}\right) \beta_{A}\left(\bar{p}_{A}-q_{A}\right) z^{2}+\left[q_{A} \beta_{A}+\bar{q}_{A} \alpha_{A}\right] z}{\left(1-\alpha_{A}\right)\left(1-\beta_{A}\right)\left(p_{A}-\bar{q}_{A}\right) z^{2}-\left[q_{A}\left(1-\beta_{A}\right)+p_{A}\left(1-\alpha_{A}\right)\right] z+1} .
\end{aligned}
$$

We define $D(z)$ and $S(z)$ as the generation function of $D$ and $S$, respectively. Then, we have

$$
D(z)=S(z) I(z)=\frac{a_{3} z^{3}+a_{2} z^{2}+a_{1} z}{b_{3} z^{3}+b_{2} z^{2}+b_{1} z+1}
$$

where

$$
\begin{aligned}
a_{3}= & (1-\sigma)\left(p_{A}-\bar{q}_{\boldsymbol{A}}\right)\left\{\left(1-\alpha_{\boldsymbol{A}}\right)\left[1-\beta_{\boldsymbol{A}}-P^{+}(2,0)\right]-\left(1-\beta_{\boldsymbol{A}}\right) P^{+}(1,0)\right\} \\
a_{2}= & (1-\sigma)\left\{P^{+}(2,0)\left[\left(p_{\boldsymbol{A}}+q_{\boldsymbol{A}}\right)\left(1-\boldsymbol{\alpha}_{\boldsymbol{A}}\right)+\alpha_{\boldsymbol{A}}\right]+P^{+}(1,0)\left[\left(p_{\boldsymbol{A}}+q_{\boldsymbol{A}}\right)\left(1-\beta_{\boldsymbol{A}}\right)+\beta_{\boldsymbol{A}}\right]\right\} \\
& +(1-\sigma)\left[p_{\boldsymbol{A}}\left(\boldsymbol{\alpha}_{\boldsymbol{A}}+\boldsymbol{\beta}_{\boldsymbol{A}}-1\right)-q_{\boldsymbol{A}}\right] \\
a_{1}= & (1-\sigma)\left[1-P^{+}(1,0)-P^{+}(2,0)\right] \\
b_{3}= & -\sigma\left(1-\alpha_{\boldsymbol{A}}\right)\left(1-\boldsymbol{\beta}_{\boldsymbol{A}}\right)\left(p_{\boldsymbol{A}}-\bar{q}_{\boldsymbol{A}}\right) \\
b_{2}= & \left(1-\boldsymbol{\alpha}_{\boldsymbol{A}}\right)\left(1-\boldsymbol{\beta}_{\boldsymbol{A}}\right)\left(\boldsymbol{p}_{\boldsymbol{A}}-\bar{q}_{\boldsymbol{A}}\right)+\sigma\left[q_{\boldsymbol{A}}\left(1-\beta_{\boldsymbol{A}}\right)+p_{\boldsymbol{A}}\left(1-\alpha_{\boldsymbol{A}}\right)\right] \\
b_{1}= & -\left[\sigma+q_{\boldsymbol{A}}\left(1-\boldsymbol{\beta}_{\boldsymbol{A}}\right)+\boldsymbol{p}_{\boldsymbol{A}}\left(1-\boldsymbol{\alpha}_{\boldsymbol{A}}\right)\right] .
\end{aligned}
$$

By differentiating the generating function given by (1), we can obtain the moments of the time between successive departures, the squared coefficient of variation of the interdeparture time $C_{d}^{2}$, and the throughput $\rho_{d}$.

\subsection{Autocorrelation of the Departure Process}

In this section, we obtain the autocorrelation of the interdeparture time, and the autocorrelation of the number of departures per slot. Define

$$
\begin{aligned}
D_{i j}(z) & =E\left\{z^{t_{n}}, d_{n}=j \mid d_{n-1}=i\right\} \quad \text { for } i, j=1,2, \cdots, 2 K+2 \\
D_{i}(z) & =E\left\{z^{t_{n+1}} \mid d_{n}=i\right\} \quad \text { for } i=1,2, \cdots, 2 K+2
\end{aligned}
$$


where $d_{n}$ is the state of the queue when the $n$th departure occurs, and $t_{n}$ is the time interval between the $(n-1)$ st and the $n$th departure. From the definition of $D_{i j}(z)$ and $D_{i}(z)$,

$\mathbf{C}(z)=\left|D_{i j}(z)\right|_{(2 K+2) \times(2 K+2)}=z\left(\mathbf{I}-z \mathbf{P}_{w o d}\right)^{-1} \mathbf{P}_{w d} \quad$ and $\quad \overrightarrow{\mathrm{D}}(z)=\left|D_{i}(z)\right|_{(2 K+2) \times 1}=\overrightarrow{\mathrm{I}}(z) S(z)$ w'ere $\overrightarrow{\mathbf{I}}(z)=\left[I_{1}(z), I_{2}(z), 1,1, \cdots, 1\right]^{T}$. Then, we have

$$
G_{d}\left(z_{1} z_{2}\right)=E\left\{z_{1}^{t_{n}} z_{2}^{t_{n+i}}\right\}=\overrightarrow{\mathbf{P}}^{+} \mathbf{C}\left(z_{1}\right) \mathbf{R}^{i-1} \overrightarrow{\mathbf{D}}\left(z_{2}\right)
$$

where $\mathbf{R}=\left(\mathbf{I}-\mathbf{P}_{w o d}\right)^{-1} \mathbf{P}_{w d}$. By differentiating the equation (2) with respect to $z_{1}$ and $z_{2}$, we have

$$
E\left\{t_{n} t_{n+i}\right\}=\overrightarrow{\mathbf{P}}^{+}\left(\mathbf{I}-\mathbf{P}_{w o d}\right)^{-2} \mathbf{P}_{w d} \mathbf{R}^{i-1} \overrightarrow{\mathbf{D}}^{\prime}(1)
$$

where $\vec{D}^{\prime}(1)=\left.\frac{d \vec{D}(z)}{d z}\right|_{z=1}$. The autocorrelation coefficient of the interdeparture times of an $\mathrm{MMBP} / \mathrm{Geo} / 1 / \mathrm{K}$ queue for lag $i, \psi_{d}(i)$, can now obtained from the expression:

$$
\psi_{d}(i)=\frac{E\left\{t_{n} t_{n+i}\right\}-E^{2}\left\{t_{n}\right\}}{\operatorname{Var}\left\{t_{n}\right\}} .
$$

Now, let $X_{n}$ be a random variable representing the number of departures at $n$th slot, where $X_{n}=0,1$. Then we have

$$
E\left\{X_{n}\right\}=E\left\{X_{n}^{2}\right\}=\mathbf{x} \vec{\lambda}_{d} \text { and } E\left\{X_{n} X_{n+i}\right\}=\mathbf{x} \mathbf{P}_{w d} \mathbf{P}_{d}^{i-1} \vec{\lambda}_{d}
$$

The autocorrelation coefficient of $\operatorname{lag} i, \phi_{d}(i)$ can be obtained using a similar expression as (3).

\section{Characterization of the Departure Process by two-state MMBP}

In this section, we examine an approximation model for characterizing the departure process by a two-state MMBP. This model can capture the correlation of the departure process of the queue as well as burstiness. The fitted two-state MMBP is characterized by the four parameters, $p_{\text {est }}, q_{\text {est }}, \alpha_{\text {est }}$, and $\beta_{\text {est }}$. We match $\rho_{d}, C_{d}^{2}$, and the autocorrelation coefficients 
$\phi_{d}(1)$ and $\psi_{d}(1)$ of the departure process in order to obtain $p_{\text {est }}, q_{\text {est }}, \alpha_{\text {est }}$ and $\beta_{\text {est }}$. First, we can obtain $\alpha_{\text {est }}$ from the following equation:

$$
\begin{aligned}
& \left\{4 C_{d}^{2}\left(1-\rho_{d}\right) \phi_{d}(1) \psi_{d}(1)+\left(C_{d}^{2}+\rho_{d}-1\right)\left[\left(C_{d}^{2}+\rho_{d}-1\right)-2\left(1-\rho_{d}\right) \phi_{d}(1)\right]\right\} \alpha_{e s t}^{2}- \\
& \left\{4 C_{d}^{2}\left(1-\rho_{d}^{2}\right) \phi_{d}(1) \psi_{d}(1)+\left(C_{d}^{2}+\rho_{d}-1\right)^{2} \rho_{d}+\left[\left(C_{d}^{2}-1\right)^{2}-\rho_{d}^{2}\right]\left(1-\rho_{d}\right) \phi_{d}(1)\right\} \alpha_{e s t}+ \\
& 4 C_{d}^{2}\left(1-\rho_{d}\right) \rho_{d} \phi_{d}(1) \psi_{d}(1)=0 .
\end{aligned}
$$

Then, using $\alpha_{\text {est }}, \rho_{d}, C_{d}^{2}$, and $\phi_{d}(1)$, we have

$$
\beta_{\text {est }}=\frac{\rho_{d}\left(C_{d}^{2}+\rho_{d}-1\right)\left[\left(1-\rho_{d}\right) \phi_{d}(1)-\alpha_{e s t}+\rho_{d}\right]+2\left(\alpha_{e s t}-\rho_{d}\right) \rho_{d}\left(1-\rho_{d}\right) \phi_{d}(1)}{\left(1-\rho_{d}\right) \phi_{d}(1)\left[\left(C_{d}^{2}+\rho_{d}+1\right) \alpha_{e s t}-2 \rho_{d}\right]-\left(\alpha_{e s t}-\rho_{d}\right)\left(C_{d}^{2}+\rho_{d}-1\right)} .
$$

Finally, $p_{\text {est }}$ and $q_{\text {est }}$ can be obtained from

$$
\begin{aligned}
& p_{\text {est }}=1-\frac{\left(\alpha_{e s t}-\rho_{d}\right)\left(\rho_{d}-\beta_{e s t}\right)-\rho_{d}\left(1-\rho_{d}\right) \phi_{d}(1)}{\left(\alpha_{e s t}-\beta_{e s t}\right)\left(\rho_{d}-\beta_{\text {est }}\right)} \\
& q_{\text {est }}=1-\frac{\left(\alpha_{e s t}-\rho_{d}\right)\left(\rho_{d}-\beta_{\text {est }}\right)-\rho_{d}\left(1-\rho_{d}\right) \phi_{d}(1)}{\left(\alpha_{\text {est }}-\beta_{\text {est }}\right)\left(\alpha_{\text {est }}-\rho_{d}\right)}
\end{aligned}
$$

It is possible to have two roots for $\alpha_{\text {est }}$. But, one root has the same value as $\beta_{\text {est }}$. We note that there is no guarantee that we will obtain a feasible solution. If the solution is not feasible, we can obtain a feasible set of $\left(p_{\text {est }}, q_{\text {est }}, \alpha_{\text {est }}, \beta_{\text {est }}\right)$ as follows: Let $\psi_{\text {est }}(i)$ be the autocorrelation coefficient of the interdeparture time for lag $i$ estimated using the fitted two-state MMBP. Define

$$
\epsilon_{\psi}(k)=\sum_{i=1}^{k}\left|\psi_{d}(i)-\psi_{e s t}(i)\right|
$$

Then we have:

step $0:$ Set $\alpha=0, \delta=1.0 E-6$, and $\epsilon_{\psi}^{(0)}=1.0 E+30$.

step 1: Set $\alpha=\alpha+\delta$. If $\alpha>1$, stop and choose $p_{\text {est }}^{(0)}, q_{\text {est }}^{(0)}, \alpha_{\text {est }}^{(0)}$, and $\beta_{\text {est }}^{(0)}$ for parameters of the fitted two-state MMBP.

step 2: Calculate $p_{\text {est }}, q_{\text {est }}$, and $\beta_{\text {est }}$ using equations (5), (6), and (4) by setting $\alpha_{\text {est }}$ $=\alpha$. If feasible go to step 3 , else go to step 1

step 3: Compute $\psi_{\text {est }}(1)$ and calculate $\epsilon_{\psi}^{(1)}=\epsilon_{\psi}(1)$. If $\epsilon_{\psi}^{(0)}>\epsilon_{\psi}^{(1)}$, reset $\epsilon_{\psi}^{(0)}=\epsilon_{\psi}^{(1)}, p_{e s t}^{(0)}=$ $p_{\text {est }}, q_{\text {est }}^{(0)}=q_{\text {est }}, \alpha_{\text {est }}^{(0)}=\alpha_{\text {est }}$, and $\beta_{\text {est }}^{(0)}=\beta_{\text {est }}$. Go to step 1

Extensive tests were carried out to establish the accuracy of the fitting model. Below, we present 9 different cases, each case corresponding to a departure process with different $\rho_{d}$ and $C_{d}^{2}$. In particular, we have classified the values for $\rho_{d}$ and $C_{d}^{2}$ in low, medium, and large. Each case represents a different combination of values of $\rho_{d}$ and $C_{d}^{2}$. The parameters 
of the arrival process $\left(p_{A}, q_{A}, \alpha_{A}, \beta_{A}\right), \sigma$, and buffer capacity $\mathrm{K}$ of the queue under study are given in Table 1 , and the resulting $\rho_{d}, C_{d}^{2}, \psi_{d}(1)$, and $\phi_{d}(1)$ for each case are given in Table 2. Define

$$
\epsilon_{\phi}(k)=\sum_{i=1}^{k}\left|\phi_{d}(i)-\phi_{\text {est }}(i)\right| \text { and } \epsilon_{D}(k)=\sum_{i=1}^{k}\left|P_{\text {exact }}\{D=i\}-P_{\text {eet }}\{D=i\}\right|
$$

where $\phi_{\text {eat }}(i)$ is the estimated autocorrelation coefficient of the number of departures for $\operatorname{lag} i, P_{\text {exact }}\{D=i\}$ is the exact probability that the interdeparture time is $i$ slot(s), and $P_{\text {est }}\{D=i\}$ is the estimated probability that the interdeparture time is $i \operatorname{slot}(\mathrm{s})$. In Table 2, we give the observed values for $\epsilon_{\psi}(5000), \epsilon_{\phi}(5000)$, and $\epsilon_{D}(5000)$ for each of the 9 cases. From Table 2, we see that the fitted MMBP tracks well the interdeparture time distribution and two autocorrelation functions.

\section{Analysis of a Tandem Configuration with Customer Loss}

In this section, we analyze approximately a tandem configuration of discrete-time finite capacity queues using the model for fitting a two-state MMBP described in the previous section. Let us consider an open queueing network consisting of $N$ nodes linked in tandem as shown in figure 1 . We assume that each node has a finite capacity. Let $K_{i}$ be the maximum capacity of node $i, i=1,2, \cdots, N$. A customer enters a node if it arrives at a time when the node is not full. Otherwise, it gets lost. The arrival process to the first node is assumed to be a two-state MMBP. For each node $i$, the service time is assumed to be geometrically distributed with probability $\sigma_{i}$. We note that the $N$ servers are not synchronized. That is, the service slots of a server begin at a different time than the service slots of the other servers. However, all service slots of all servers are equal. Finally, we assume that customers in a node are served in a FIFO manner.

The approximation algorithm decomposes the queueing network into individual nodes, and each node is analyzed in isolation. Let us consider node $i$. We can obtain the generating function and autocorrelation coefficients of the departure process of node $i$ using equations (1), and the results in section 3.2. Using the fitting model mentioned above, we 


\begin{tabular}{|c||l|l|l|l||c|c|}
\hline \multicolumn{1}{|c||}{} & \multicolumn{4}{c||}{ Arrival Process } & \multicolumn{2}{c|}{ Node } \\
\cline { 2 - 7 } & \multicolumn{1}{|c|}{$p_{\boldsymbol{A}}$} & \multicolumn{1}{c|}{$q_{\boldsymbol{A}}$} & \multicolumn{1}{c|}{$\boldsymbol{\alpha}_{\boldsymbol{A}}$} & \multicolumn{1}{c|}{$\beta_{\boldsymbol{A}}$} & $\sigma$ & $\mathrm{K}$ \\
\hline \hline 1 & 0.65 & 0.7 & 0.11 & 0.06 & 0.2 & 4 \\
\hline 2 & 0.9995 & 0.9999 & 0.5 & 0.01 & 0.1 & 4 \\
\hline 3 & 0.9999 & 0.99992 & 0.223 & 0.001 & 0.1 & 4 \\
\hline 4 & 0.85 & 0.74 & 0.6 & 0.45 & 0.1 & 8 \\
\hline 5 & 0.999687 & 0.999367 & 0.8295915 & 0.01259 & 0.1 & 8 \\
\hline 6 & 0.9999 & 0.9998 & 1.0 & 0.003 & 0.2 & 8 \\
\hline 7 & 0.99 & 0.8 & 0.95 & 0.5 & 0.1 & 16 \\
\hline 8 & 0.9999 & 0.995 & 0.9 & 0.01 & 0.1 & 16 \\
\hline 9 & 0.999999 & 0.99999 & 1.0 & 0.008 & 0.1 & 32 \\
\hline
\end{tabular}

Table 1: The parameters of arrival process and node

\begin{tabular}{|c||c|c|c|c|c|}
\hline \multicolumn{1}{|c||}{} & \multicolumn{5}{c|}{ Departure Process } \\
\cline { 2 - 6 } No & $\rho_{d}$ & $C_{d}^{2}$ & $\psi_{d}(1) / \epsilon_{\psi}(5000)$ & $\phi_{d}(1) / \epsilon_{\phi}(5000)$ & $\epsilon_{D}(5000)$ \\
\hline \hline 1 & $8.3077 \mathrm{e}-2$ & $9.2452 \mathrm{e}-1$ & $2.2539 \mathrm{e}-4 / 4.8091 \mathrm{e}-6$ & $2.4030 \mathrm{e}-3 / 1.3806 \mathrm{e}-4$ & $2.0866 \mathrm{e}-4$ \\
\hline 2 & $9.1639 \mathrm{e}-2$ & $1.4097 \mathrm{e}+1$ & $4.6269 \mathrm{e}-1 / 2.4312 \mathrm{e}-2$ & $3.9990 \mathrm{e}-1 / 3.8147 \mathrm{e}-1$ & $2.9991 \mathrm{e}-4$ \\
\hline 3 & $9.9666 \mathrm{e}-2$ & $1.0191 \mathrm{e}+2$ & $4.5868 \mathrm{e}-1 / 1.7310 \mathrm{e}-4$ & $1.3558 \mathrm{e}-1 / 1.3194 \mathrm{e}-2$ & $2.4668 \mathrm{e}-5$ \\
\hline 4 & $5.4512 \mathrm{e}-1$ & $4.6956 \mathrm{e}-1$ & $6.8816 \mathrm{e}-3 / 1.4186 \mathrm{e}-3$ & $1.0621 \mathrm{e}-2 / 1.4761 \mathrm{e}-3$ & $2.5340 \mathrm{e}-4$ \\
\hline 5 & $5.5873 \mathrm{e}-1$ & $2.7325 \mathrm{e}+1$ & $4.6822 \mathrm{e}-1 / 5.1345 \mathrm{e}-3$ & $5.9673 \mathrm{e}-1 / 7.7976 \mathrm{e}-1$ & $1.7401 \mathrm{e}-4$ \\
\hline 6 & $5.3476 \mathrm{e}-1$ & $1.1064 \mathrm{e}+2$ & $4.6671 \mathrm{e}-1 / 5.5254 \mathrm{e}-3$ & $5.6671 \mathrm{e}-1 / 5.4205 \mathrm{e}-1$ & $1.1340 \mathrm{e}-12$ \\
\hline 7 & $8.9905 \mathrm{e}-1$ & $1.0164 \mathrm{e}-1$ & $2.2076 \mathrm{e}-3 / 2.4949 \mathrm{e}-2$ & $2.0006 \mathrm{e}-3 / 2.4954 \mathrm{e}-2$ & $9.1729 \mathrm{e}-6$ \\
\hline 8 & $8.7698 \mathrm{e}-1$ & $2.2749 \mathrm{e}+0$ & $3.1496 \mathrm{e}-1 / 2.6812 \mathrm{e}-2$ & $1.3288 \mathrm{e}-1 / 4.9581 \mathrm{e}-1$ & $8.2126 \mathrm{e}-5$ \\
\hline 9 & $8.1894 \mathrm{e}-1$ & $1.8419 \mathrm{e}+1$ & $4.9447 \mathrm{e}-1 / 9.6863 \mathrm{e}-3$ & $4.4333 \mathrm{e}-1 / 1.2827 \mathrm{e}-3$ & $4.5069 \mathrm{e}-11$ \\
\hline
\end{tabular}

Table 2: The validation results

can then obtain the set of parameters $\{p, q, \alpha, \beta\}$ of a two-state MMBP which characterizes approximately the departure process of node $i$. This MMBP becomes the arrival process to the next down-stream node $i+1$. Since the arrival process to node $i+1$ is modeled as a two-state MMBP, node $i+1$ can also be analyzed as an MMBP/Geo/1/Ki+1 queue. In this manner, we can analyze all queues individually starting from the first node and proceeding sequentially to the last node.

The accuracy of the approximation algorithm was tested by comparing the approximate results against simulation data. We present two different examples involving a 10 node tandem configuration. The first example corresponds to a case of a strongly correlated input 
traffic to the first node and the second example to a case of a less correlated input traffic. The input traffic for both examples has the same $\rho_{A}$ and $C_{A}^{3}$. The parameters of the arrival process to the first node for example 1 are: $p_{A}=0.9993135, q_{A}=0.9987141, \alpha_{A}=0.7585780$, and $\beta_{A}=0.01563149$ (thus resulting to a $\rho_{A}=0.5, C_{A}^{2}=20.0, \psi_{A}(1)=0.45$, and $\phi_{A}(1)=0.5$ ).

For example 2, $\boldsymbol{p}_{\boldsymbol{A}}=0.9996268, q_{A}=0.9962970, \alpha_{A}=0.5503012$, and $\beta_{A}=0.0009596972$ (thus resulting to a $\psi_{A}(1)=0.1$ and $\phi_{A}(1)=0.1$ ). In both examples the values of $K_{i}$ and $\sigma_{i}$ are: $K_{i}=8$ for $i=1, \cdots, 10, \sigma_{i}=0.1$ for $i=2,4,6,8,10$, and $\sigma_{i}=0.2$ for $i=1,3,5,7,9$. The approximation results were shown in Figures 2-5. In particular, figures 2-3 are for example 1 , and figures 4-5 for example 2. In Figure 2 we give the queue length distribution for nodes 2, 5, and 10, the $\operatorname{Pr}\{$ empty $\}, \operatorname{Pr}\{$ full $\}$, and customer loss for each queue. In Figure 3 we give the squared coefficient of variation of the interdeparture time and the autocorrelation coefficient of lag 1 for each node. The approximate results for example 2 are presented in the same way as in example 1 in Figures 4-5. (We note that the confidence intervals in figures 2 and 4 were not plotted as they were extremely small.) In general, the decomposition algorithm was found to have a good accuracy.

\section{Conclusion}

The main contribution of this paper is a simple and accurate method for charaterizing the output of a two-state MMBP/Geo/1/K queue by a two-state MMBP. This type of distribution captures the notion of burstiness and the correlation of successive interarrival times, which are two important features of the anticipated traffic on future high-speed networks. 


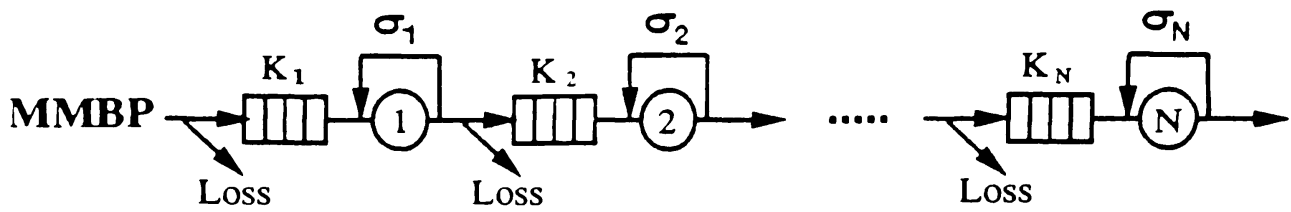

Figure 1: An open tandem queueing network under investigation
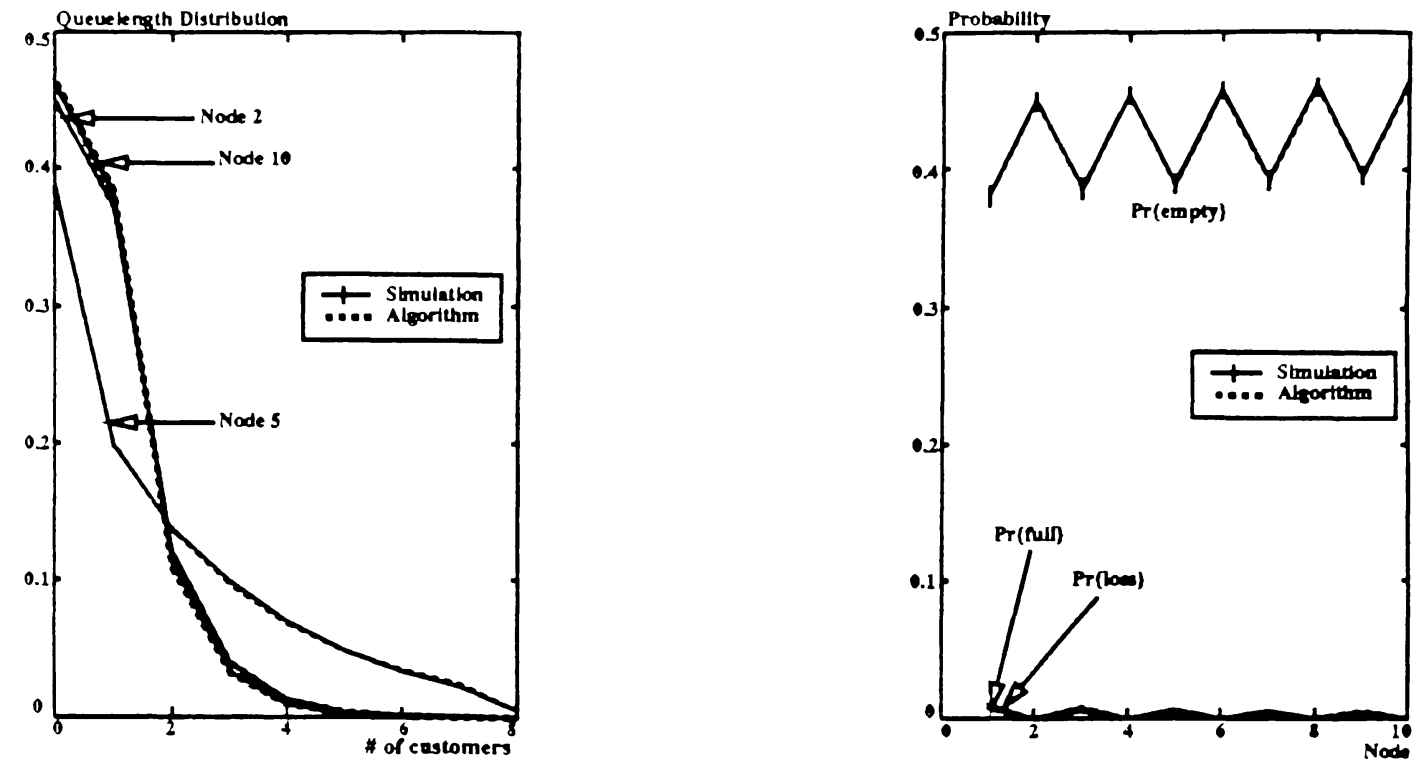

Figure 2: QLD and $P(0), P(K)$, and $P\{$ loss $\}$ (example 1)
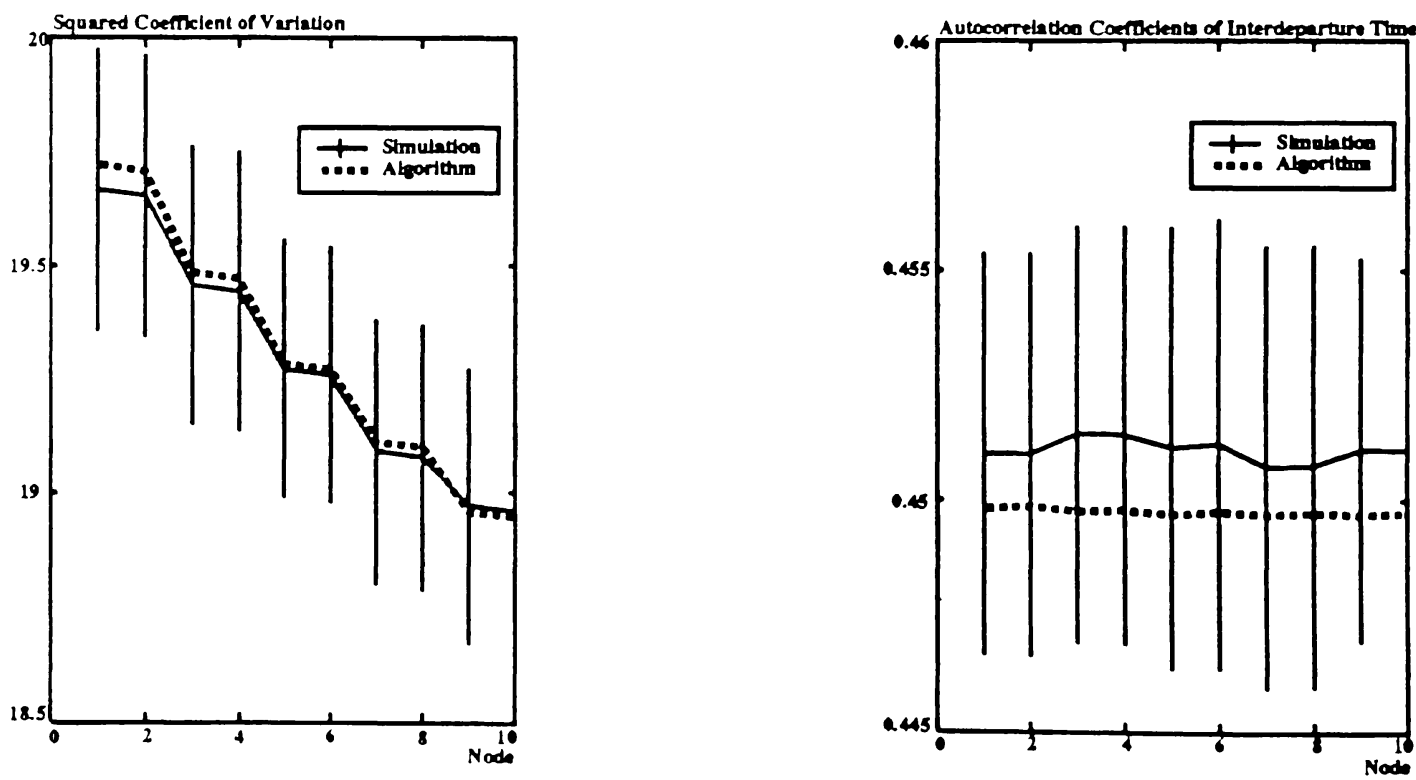

Figure 3: $C_{d}^{2}$ and $\psi_{d}(1)$ (example 1) 

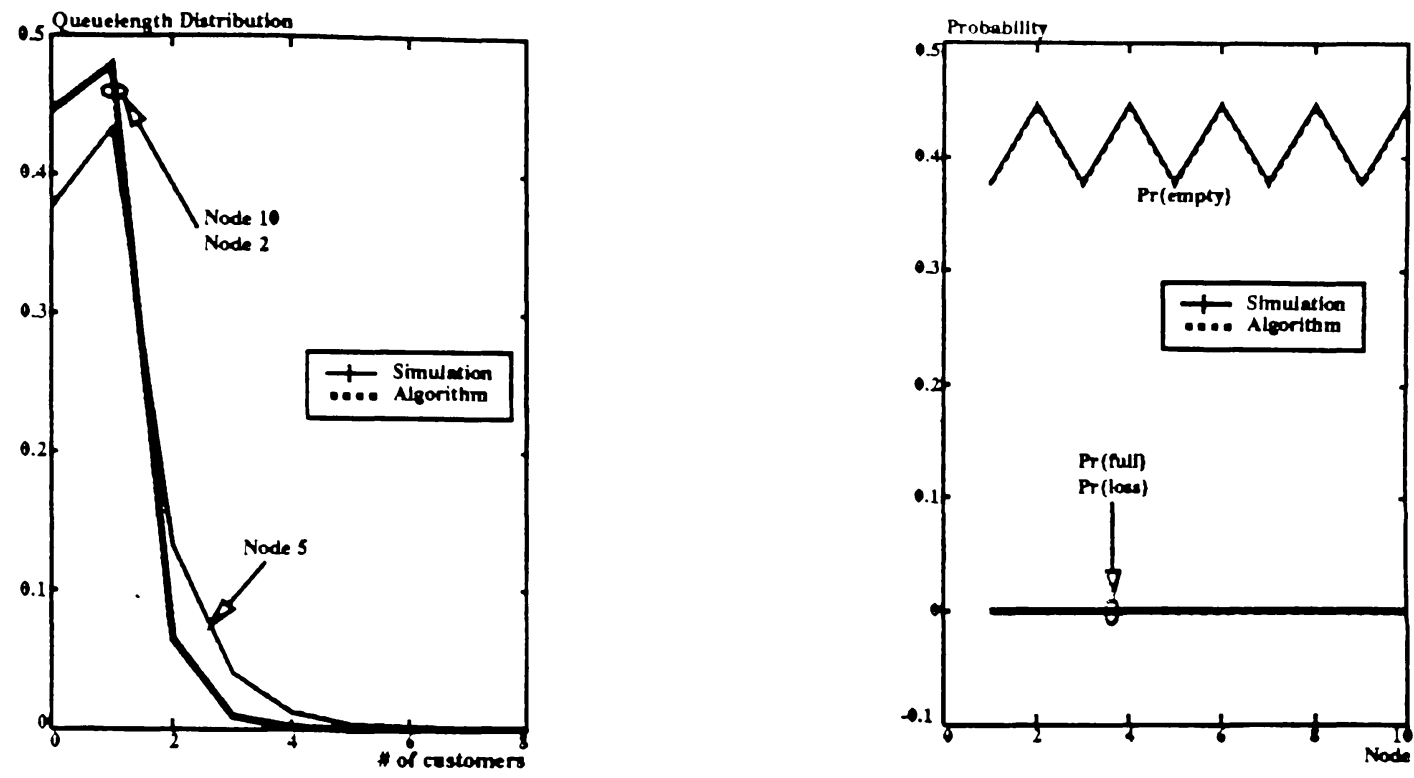

Figure 4: QLD and $P(0), P(K)$, and $P\{$ loss $\}$ (example 2)
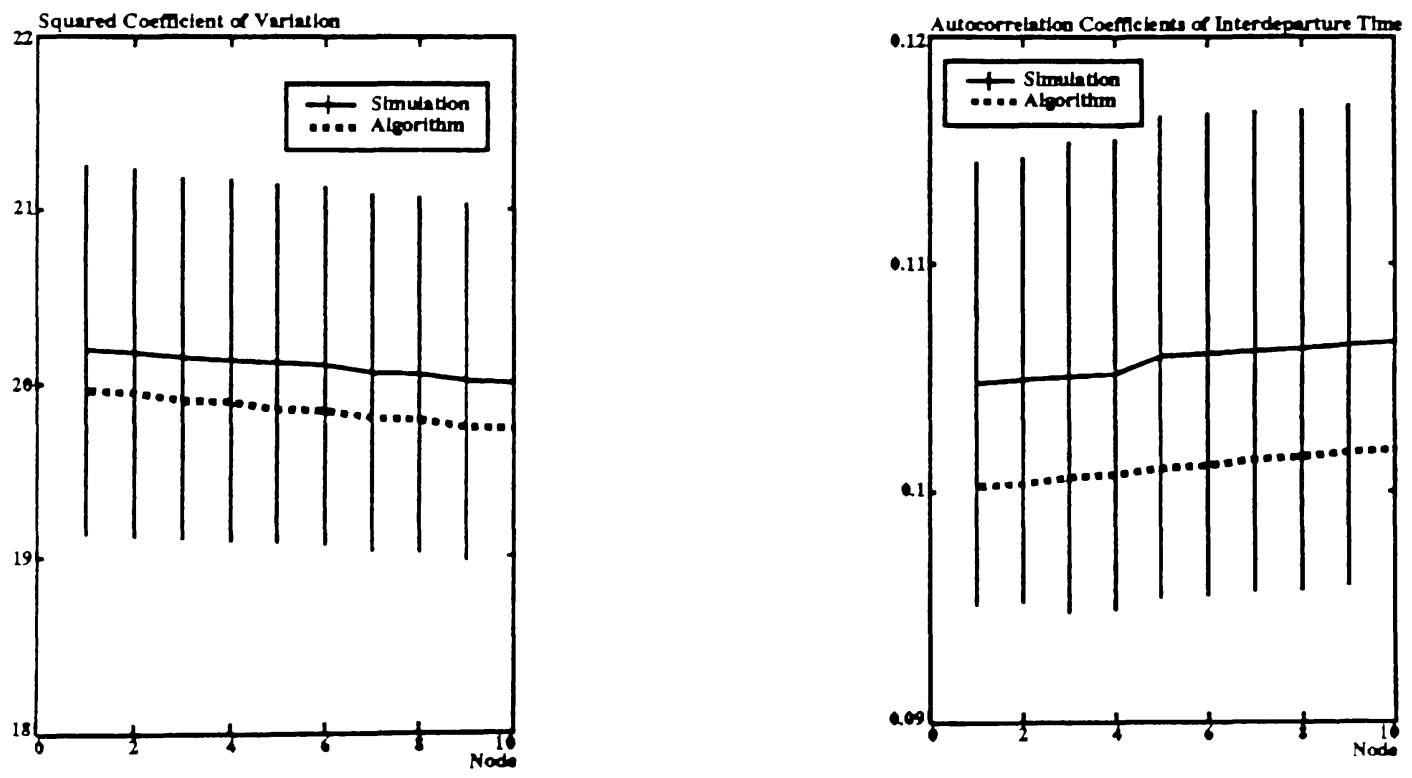

Figure 5: $C_{d}^{2}$ and $\psi_{d}(1)$ (example 2) 


\section{References}

[1] G. Pujolle and H. G. Perros, "Queueing systems for modelling ATM networks," in Proc. of the Int'l. Conf. on the Performance of Distributed Systems and Integrated Comm. Networks, pp. 10-12, Kyoto, Japan, Sept. 1991.

[2] D. Park and H. G. Perros. Approximate analysis of discrete-time tandem queueing networks with customer loss. Technical report, Computer Science, North Carolina State University, Raleigh, NC, 1991.

[3] K. Meier-Hellstern, "A fitting algorithm for Markov-modulated Poisson process having two arrival rates," E.J.O.R., vol. 29, pp. 370-377, 1987.

[4] P. Tran-Gia, "Discrete time analysis for the interdeparture distribution of GI/G/1 queue," in Teletraffic Analysis and Computer Performance Evaluation, pp. 341-357. O. J. Boxma, J. W. Cohen, and H.C. Tijms, Eds. North Holland:Elsevier Science, 1986.

[5] Y. Ohba, M. Murata, and H. Miyahara, "Analysis of interdeparture process for bursty traffic in ATM networks," IEEE J. Select. Areas Commun., no. 3, pp. 468-476, Apr. 1991.

[6] J. Morrison, "Two discrete-time queues in tandem," IEEE Trans. Commun., no. 3, pp. 563-573, Mar. 1979.

[7] J. Hsu and P. Burke, "Behavior of tandem buffers with geometric input and Markovian output," IEEE Trans. Commun., pp. 358-361, Mar. 1976.

[8] G. Pujolle, "Multiclass distrete-time queueing systems with a product form solution," in Int'l. Seminar on the Performance of Distributed and Parallel Systems, pp. 261-270, Sept. 1991.

[9] A. Bhargava, J. Kurose, D. Towsley, and G. Vanleemput, "Performance comparison of error control schemes in high-speed computer communication network," IEEE J. Select. Areas Commun., no. 9, pp. 1565-1575, Dec. 1988.

[10] P. P. Bocharov and F. K. Albores, "On two-stage exponential queueing system with internal losses or blocking," Prob. Control Inform. Theory, pp. 365-379, 1980.

[11] T. D. Morris and H. G. Perros, "Performance analysis of a multi-buffered Banyan ATM switch under bursty traffic," in INFOCOM '92, pp. 436-445, Florence, Italy, May 1992.

[12] A. Nilsson, Private Communication. North Carolina State University, Raleigh, NC.

[13] O. Hashida, Y. Takahashi, and S. Shimogawa, "Switched Batch Bernoulli Process(SBBP) and the discrete-time SBBP/G/1 queue with application to statistical multiplexer performance," IEEE J. Select. Areas Commun., no. 3, Apr. 1991. 